\title{
The role of peripheral retinal angiography in the diagnosis of adult Coats' disease based on a case report
}

\author{
Monika Turczyńska, Joanna Brydak-Godowska \\ Department of Ophthalmology, Infant Jesus Clinical Hospital, University Clinical Center, Medical University of Warsaw, Warsaw, Poland
}

\begin{abstract}
The paper presents a case of a 58-year-old woman with hypertension, who suffered unilateral visual loss due to vitreous hemorrhage in the right eye. Clinical examination, fluorescein and indocyanine green angiography and ultrasonography were performed.

Following vitreous hemorrhage resorption, fundoscopy showed atypical retinal vessels located temporally to the macula. Fluorescein and indocyanine green angiograms confirmed atypical vessels in the peripheral retina.

Diagnosis of adult onset Coats' disease was made for the atypical lesions and treatment with retinal laser photocoagulation was administered to prevent further hemorrhage.
\end{abstract}

Coats' disease, most commonly seen in male children, is rarely diagnosed in the adult female population. In adults, the course of the disease tends to be less severe than in children. When investigating the cause of vitreous hemorrhage, we have to take pathological neovascularization typical for Coat's disease into consideration as a differential diagnosis. Fundoscopy of the peripheral retina is an examination that can aid in diagnosis after absorption of vitreous hemorrhage. Wide-field angiography confirms the diagnosis, enables precise localization of pathological vessels and their activity, and helps determine treatment effectiveness.

KEY WORDS: peripheral retinal angiography, wide-field angiography, vascular malformations, Coats' disease, Leber's miliary aneurysms, vitreous hemorrhage.

\section{INTRODUCTION}

Congenital retinal telangiectasias may involve the macular area or the peripheral part of the retina. Peripheral retinal telangiectasia occurring in children is commonly referred to as Coats' disease, named for George Coats who first identified the condition in 1908 [1]. The etiology of the disease remains unknown. In the majority of cases, the disorder affects only one eye, and occurs predominantly in male children [2]. The presentation leading to diagnosis typically includes decreased visual acuity (43\%), strabismus (23\%), leukocoria (20\%), pain (6\%), heterochromia and nystagmus (1\%), but the disease can also be asymptomatic (8\%) [3].

Similar unilateral vascular malformations involving the peripheral retina in adult men were described by Theodor Leber in 1912 [4]. The disease is known as Leber's miliary aneurysms. It was not until 1955 that Reese elucidated similarities between both diseases, and proposed that Leber's miliary aneurysms were a milder form of Coats' disease [5].

A characteristic feature of these conditions is the presence of pathologically altered, tortuous, enlarged vascular loops with aneurysmal dilations (micro- and macroaneurysms).
The malformations involve small and medium-sized blood vessels [3]. The onset of the disease is asymptomatic, but over time exudative lesions appear, including macular edema, hard exudates, ecchymosis and hemorrhages. The younger the patient, the more severe the course of the disease and the poorer the prognosis. In children, exudative retinal detachment and a permanent reduction in visual acuity are more prevalent [6]. In this age group, the manifestations of Coats' disease should always be differentiated from retinoblastoma [3].

In adults, the disease may be asymptomatic for a relatively long period. Visual acuity deteriorates when the macula is affected by exudative lesions. Occasionally, pathologically altered vascular loops are discovered on routine eye examinations, for example during periodic workplace health checks. At times, the first sign of the disease is intravitreal hemorrhage.

\section{CASE REPORT}

In October 2005, a 58-year-old woman was referred to the Department of Ophthalmology at the Infant Jesus Clinical Hospital in Warsaw on an emergency basis following a sud- 


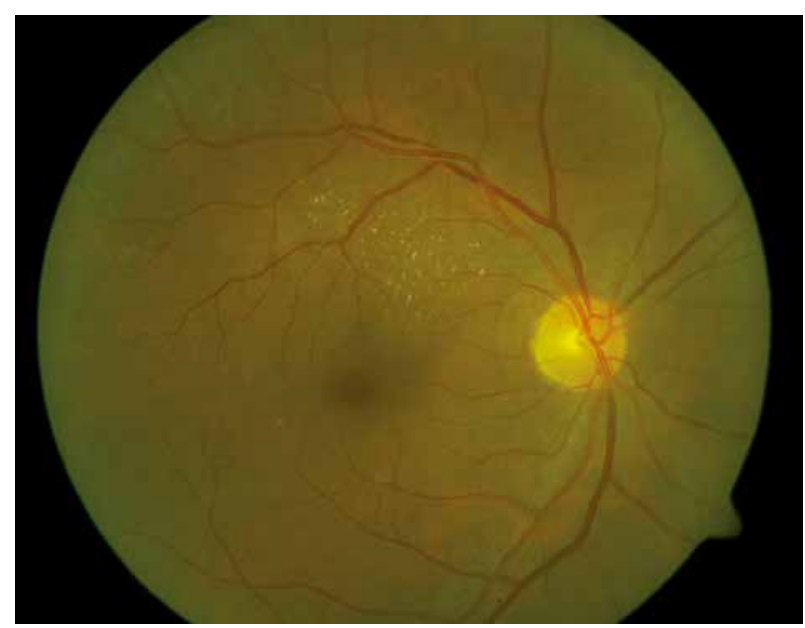

Figure 1. Color image of the posterior pole of the right eye with diffuse hard exudates in the superior region of the macula. Visible signs of compression of superior temporal vessels at the arteriovenous junctions

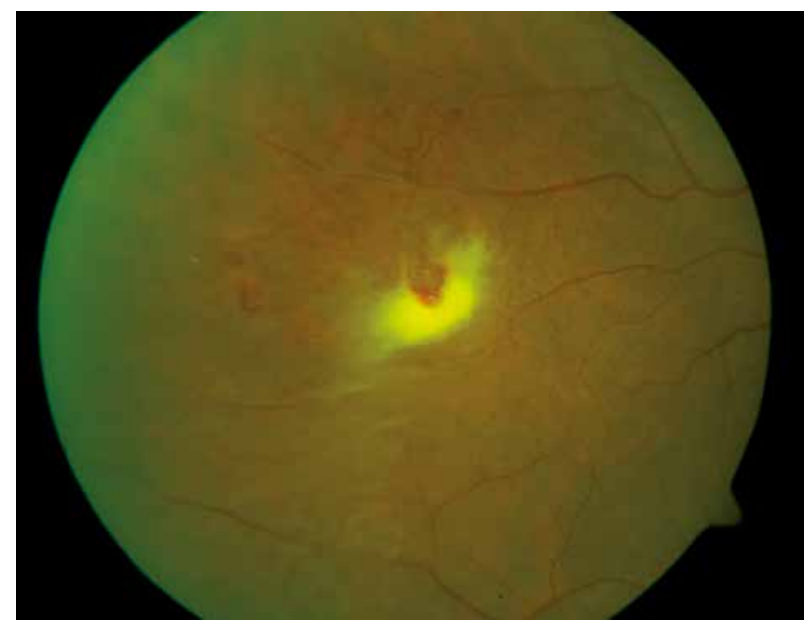

Figure 2. Color image of the right eye temporal peripheral retina with pathological vascular loops and absorbing hemorrhage before laser photocoagulation

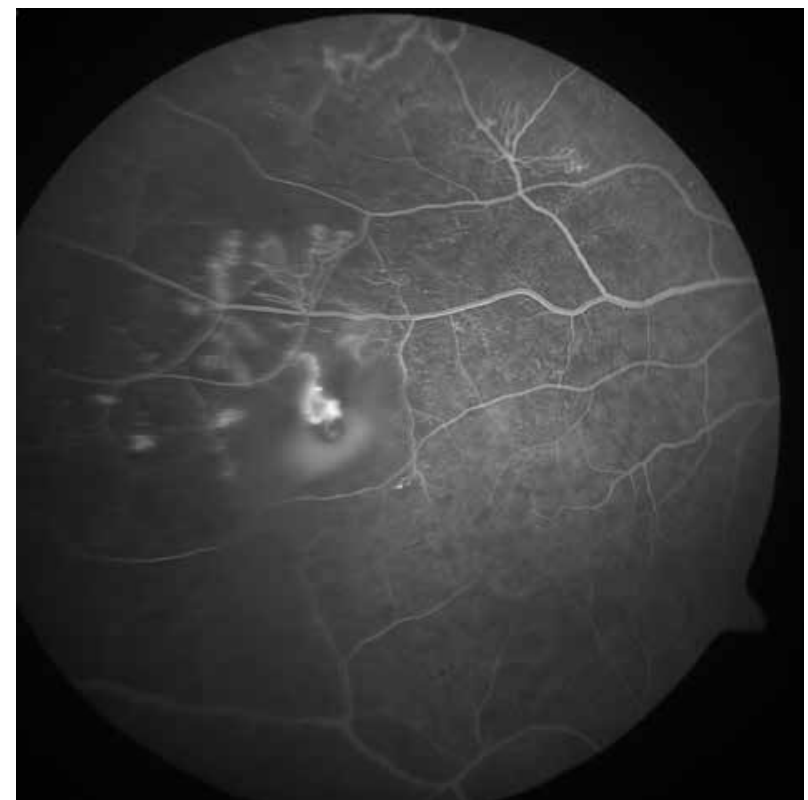

Figure 3. Fluorescein angiogram (early phase) of the temporal peripheral retina of the right eye with pathological vascularization located mainly venously with ischemic areas between vascular loops den decrease in visual acuity in the right eye (RE). On admission, the ophthalmologic examination revealed visual acuity decreased to $1 / 10$ (no improvement after correction), normal anterior segment, and massive vitreous hemorrhage preventing funduscopic examination in the RE. In the left eye (LE), the following findings were made: best corrected visual acuity (BCVA) 8/10, normal anterior segment, narrowing of arterial and venous vessels in the eye fundus with signs of compression at arteriovenous junctions. The features were consistent with hypertensive angiopathy grade 2 . The blood pressure on admission was $160 / 110 \mathrm{mmHg}$. The patient's medical history revealed a head injury sustained approximately a month before the onset of symptoms. The woman did not seek medical advice because she experienced no symptoms. She also stated that she had not previously had any ophthalmological check-ups, and denied any problems with her eyesight. She received treatment for arterial hypertension and kidney stones. Because of the patient's history of head injury, a retinal tear was suspected. An ultrasound examination was performed, but apart from vitreous hemorrhage, no pathologies were found, and the retina was normally attached. Treatment with etamsylate, rutoside and ascorbic acid was started to accelerate the resorption of extravasated blood. The patient's blood pressure was normalized.

Two months later, an ophthalmologic examination revealed full visual acuity for far and near vision in the RE. Indirect ophthalmoscopy identified features of hypertensive angiopathy grade 2 , and small hard exudates in the upper part of the macula (Figure 1). A conglomerate of an organizing blood clot and abnormal vascular malformations forming loops were observed at the temporal periphery of the retina (Figure 2). The diagnostic work-up was extended to include fluorescein angiography (FA), followed by indocyanine green angiography (ICGA), with a special focus on the evaluation of changes in the temporal peripheral retina. Angiographic examinations were performed with the Zeiss FF450 Fundus Camera. The maximum field of imaging in this system is $50^{\circ}$, and this field angle was set to visualize the periphery of the retina after appropriate eye positioning. Fluorescein angiography examination of the RE found no abnormalities in the macular angiograms. Autofluorescence attributable to an organizing blood clot was shown within the temporal periphery, in the prechoroidal phase. Following dye administration, pathologically altered vascular loops were revealed, primarily on the venous vessels, with leakage of the dye through vascular walls at later examination stages. Areas devoid of capillary circulation were seen between the pathologically altered blood vessels (Figure 3).

Indocyanine green angiography examination showed an area of hypofluorescence in the peripheral temporal region of the retina in the RE. The finding was consistent with an absorbing hemorrhage. In the upper part of this area, a tortuous vascular loop was visible, with local vascular wall dilation (an aneurysm) and stagnation of the dye at the site of the aneurysm until the late examination stages (Figure 4). Based on the examination results thus obtained, the diagnosis of adultonset Coats' disease was made. 
Treatment by laser photocoagulation (LPC) around the vascular loops was initiated to separate pathological vascularization from the posterior pole of the eye (Figure 5).

After a few weeks, the hard exudates in the macular area were found to have resolved, and further resorption of the peripheral hemorrhage was noted. Follow-up FA examination was performed (Figure 6). Over the next two years, the patient reported regularly for follow-up examinations. The visual acuity in the RE remained at 10/10, and there were no LPC-related complications or further haemorrhages. A follow-up visual field test performed with the oculus system demonstrated defects in peripheral vision in the RE on the nasal side. The findings correlated with the presence of vascular malformations located in the temporal peripheral retina (Figure 7).

Over the subsequent years, the patient had annual followup examinations. In the final assessment, in 2019, full visual acuity was confirmed in the RE, and pathological vascular loops, sealed by LPC, were identified at the temporal periphery of the fundus. A color fundus photograph was taken with a scanning laser ophthalmoscope (Optos 200Tx) (Figure 8) and follow-up FA examination was performed using two Optos 200Tx units (Figure 9) and Spectralis HRA + OCT (Spectralis Heidelberg Retina Angiograph + Optical Coherence Tomography) system with the aid of a wide-angle lens suitable for performing imaging examinations in a $102^{\circ}$ field of view (Figure 10). With good patient cooperation and appropriate eye positioning, the above units can be used for visualizing the extreme retinal periphery (ultra-widefield angiography - UWFA). The evaluation showed fewer pathologically altered vessels compared to the previous FA examination (2005), which still showed dye leakage indicating their abnormal structure. In consideration of the fact that the patient's ophthalmic status was stable, it was decided that she did not require further LPC. She remains under follow-up in the Department.

\section{DISCUSSION}

Coats' disease occurs in children, and usually affects one eye. The adult form of Coats's disease is referred to as Leber's miliary aneurysms (adult-onset Coats' disease). It is characterized by microangiopathies, typically located peripherally, on the temporal side of the fundus, with the presence of telangiectasias and macro- and microaneurysms [3]. The etiology of the disorder remains elusive. Coats' disease does not involve any systemic symptoms, and has no hereditary background. It occurs primarily among Caucasians and mostly affects men [2], though isolated cases of the disorder occurring in adult women have also been reported $[7,8]$.

In our reported patient, the first manifestation of the disease, in the form of vitreous hemorrhage, occurred at the age of 58 years. According to Shields et al., vitreous hemorrhage is rarely the first symptom of Coats' disease, and their analysis of 158 eyes affected by the condition revealed no such cases [3].

In adults, other causes of vitreous hemorrhage, especially those accompanied by exudative lesions, should be ruled out.

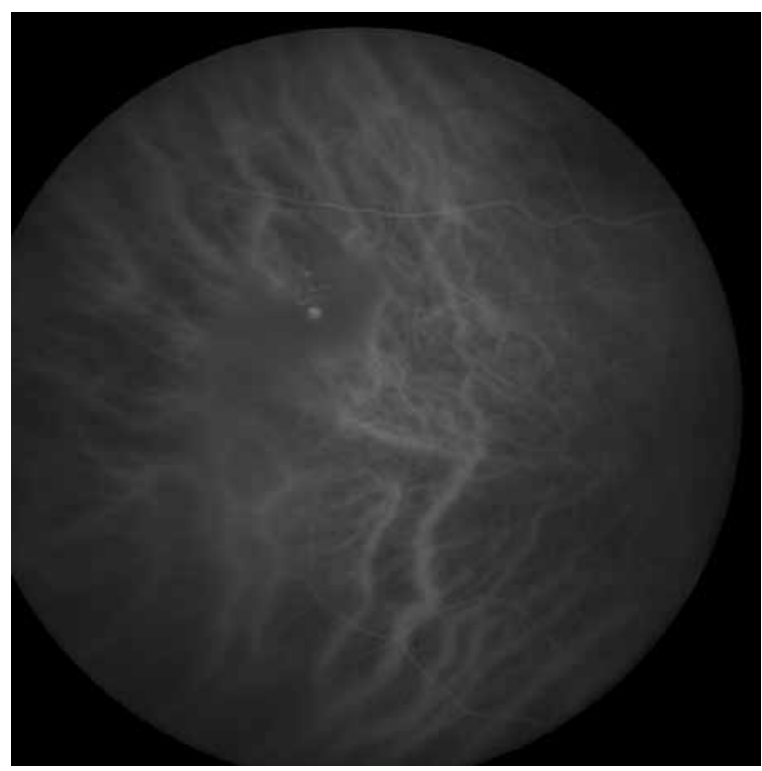

Figure 4. Indocyanine green angiogram of the temporal periphery retina of the right eye with pathological vascular loops and aneurysm

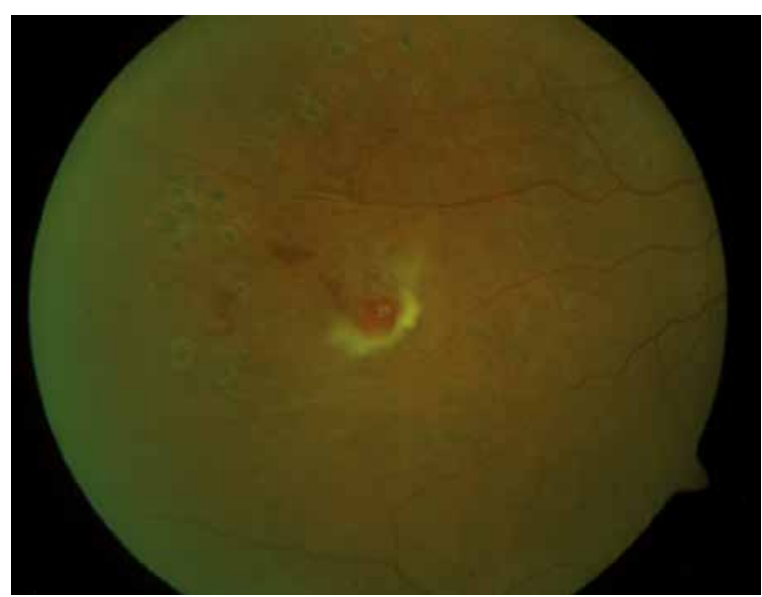

Figure 5. Color image of the temporal peripheral retina of the right eye with pathological vascular loops after laser photocoagulation

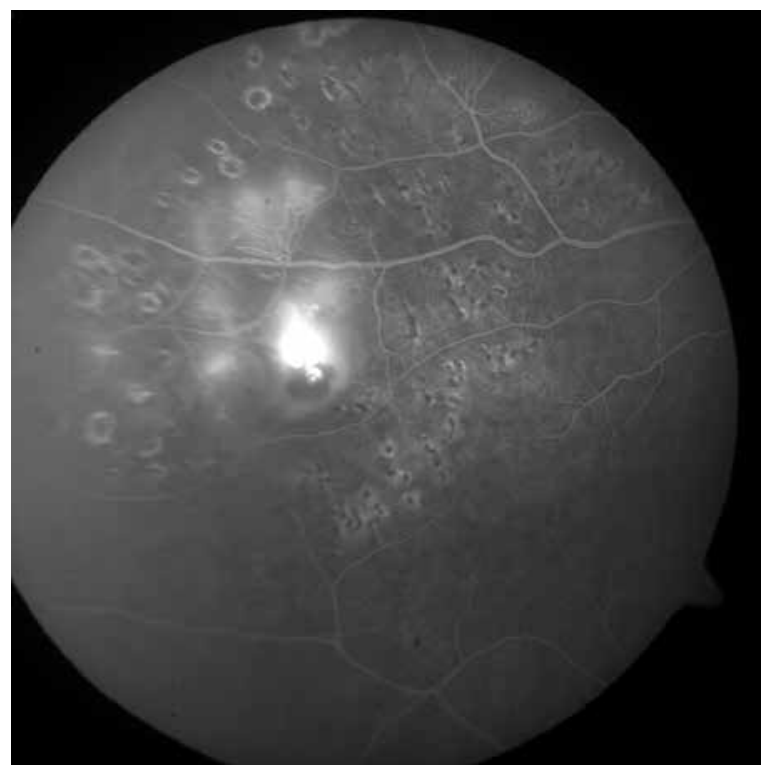

Figure 6. Fluorescein angiogram of the temporal peripheral retina of the right eye with pathological vascular loops after laser photocoagulation 


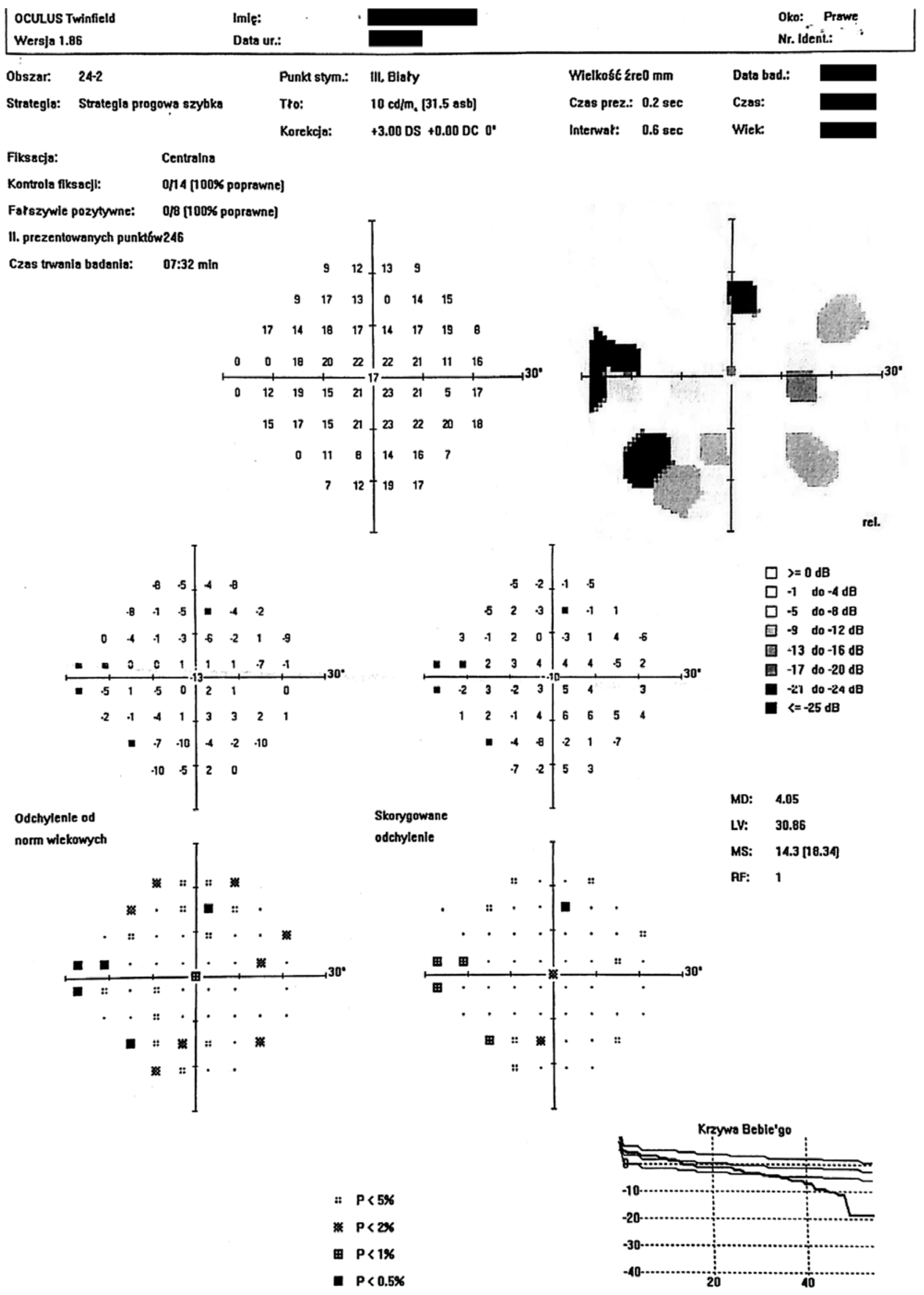

Figure 7. Perimetry of the right eye with peripheral nasal defects correlating with temporal vascular malformations 
Telangiectasias secondary to Coats' disease and the associated vitreous hemorrhaging in adults should be differentiated from vascular diseases with a higher prevalence in the population, including diabetic retinopathy or retinal venous thrombosis, but also less common pathologies such as radiation retinopathy, von Hippel-Lindau disease, retinal angiomatosis, tumors with their own vasculature, hemangiomas, sickle cell retinopathy, and retinal macroaneurysm, which most commonly involve the major retinal arteries $[9,10]$. The differential diagnosis of adult-onset Coats' disease should also comprise inflammatory vascular diseases, e.g. secondary to autoimmune, inflammatory or idiopathic diseases, such as Eales' disease [9]. Gathering the patient's history of concomitant systemic diseases including diabetes, neoplastic disease, systemic inflammatory diseases and arterial hypertension, which in itself can cause bleeding, is helpful in determining the underlying cause of hemorrhage. In cases involving massive vitreous hemorrhages without a possibility to examine the retina ophthalmoscopically, the first step should always be to rule out the diagnosis of retinal tear and rhegmatogenous retinal detachment. A helpful diagnostic modality is ultrasound scanning, which was performed as one of the first examinations in the presented case.

In the reported patient, an additional contributory effect of arterial hypertension on vitreous hemorrhage cannot be excluded, but according to the authors, the primary cause was established as abnormalities in the peripheral retinal vessels.

Indirect ophthalmoscopy with peripheral retinal evaluation is the primary examination in the diagnostic work-up in Coats' disease. Highly useful examinations supplementing the diagnostic work-up include widefield angiography (WFA) and UWFA which is suitable for the assessment of circulation in the peripheral part of the retina, as abnormal blood vessels are frequently located beyond the range of standard $30-50^{\circ}$ lenses. WFA is a suitable technique to corroborate the diagnosis, accurately evaluate the location and extent of telangiectasias and the course and structure of pathologically altered vessels, visualize aneurysmal dilatations, and assess the extent of vascular damage which correlates with the intensity of fluorescein leakage in WFA. In addition, widefield fluorescein angiography is able to visualize avascular areas between vascular loops which may require treatment by laser photocoagulation. In the reported patient, the aneurysm in the pathologically altered vascular loop was better visualized by ICGA than by FA, and the diagnosis of adult-onset Coats' disease (Leber's miliary aneurysms) was based on WFA.

Shields et al. report that Coats' disease is unilateral in 95\% of cases, but the introduction of widefield diagnostic imaging systems has made it possible to assess more accurately the peripheral retinal vessels, and visualize bilateral abnormalities. Based on UWFA images, Rabiolo et al. identified lesions characteristic of Coats' disease bilaterally in 2 out of 11 evaluated patients [11]. In their examination of patients performed using the RetCam System with a $130^{\circ}$ imaging field, Blair et al. identified bilateral, asymmetric vascular abnormalities in as many as 22 out of 32 patients with Coats' disease [12].

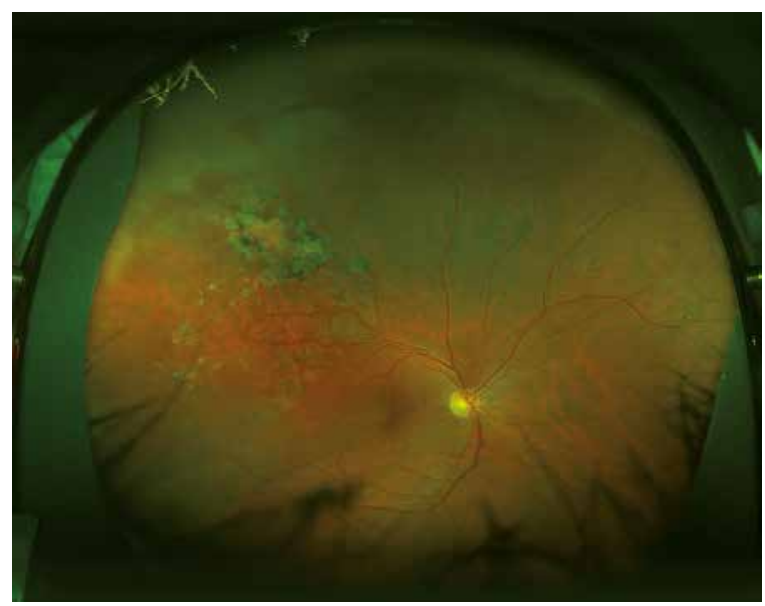

Figure 8. Color image of the right eye fundus covering the posterior pole and the peripheral retina taken with an 0ptos 200Tx system.

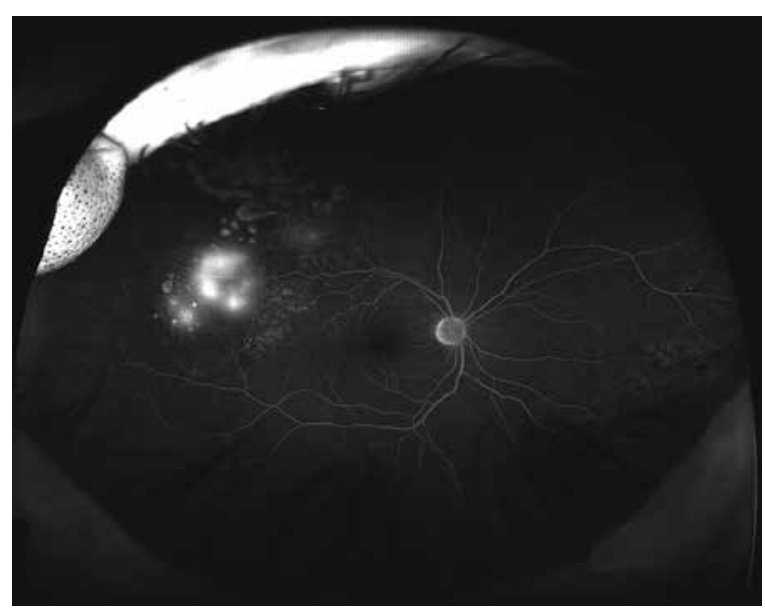

Figure 9. Angiogram of the right eye covering the posterior pole and peripheral retina demonstrating pathological vascularization with dye diffusion after laser photocoagulation. Image taken with an 0ptos 200Tx system

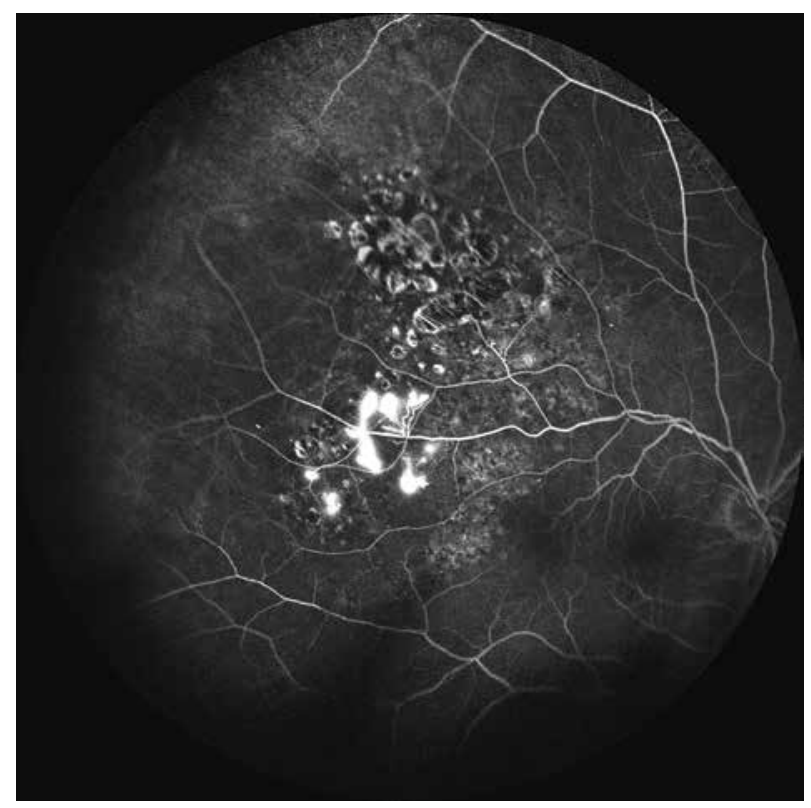

Figure 10 . Angiogram of the right eye covering the posterior pole and peripheral retina demonstrating pathological vascularization with dye diffusion, after laser photocoagulation. Image taken with the Spectralis HRA + OCT system 
In children, the lesions at the fundus at the time of diagnosis may be so advanced that the decision to initiate treatment is made on the basis of the clinical picture rather than angiography findings. Angiography in children often requires general anesthesia, though with the current technical possibilities, the examination can also be performed without it [13]. Obtaining good child cooperation is of essence in such cases. In the pediatric population, it is always important to differentiate Coats' disease from retinoblastoma. As Shields et al. report, in a group of 2,781 patients with retinoblastoma $22 \%$ of all cases were found to involve "pseudoretinoblastoma”, and patients with Coats' disease accounted for $40 \%$ of the study group [2].

Since the course of the disease in our patient was prolonged and mild, a decision was made to apply LPC to seal off the peripheral areas with pathologically altered vessels from the undamaged retina in the posterior pole. The above procedure together with laser photocoagulation of aneurysms and avascular zones are among the therapeutic modalities available for the management of milder forms of Coats' disease. Lesions located in the distal periphery can also be treated by transscleral cryotherapy. In patients with macular edema, injections of anti-VEGF agents or intravitreal steroids may be an effective adjunctive therapy $[14,15]$.

\section{CONCLUSIONS}

Adult-onset Coats' disease should be included in the differential diagnosis in adult women presenting with peripheral telangiectasias. The initial manifestation may be hemorrhage into the vitreous chamber. Coats' disease can be suspected on the basis of findings of indirect ophthalmoscopy with peripheral retinal evaluation, but pathological vascularization located around the periphery of the retina can be assessed accurately by widefield angiography. The modality plays an important role in confirming the diagnosis as well as determining the location, extent and activity of vascular pathologies. Widefield angiography is useful in the differential diagnosis, assessment of patient eligibility for treatment, and evaluation of therapeutic outcomes.

\section{DISCLOSURE}

The authors declare no conflict of interest.

\section{References}

1. Coats G. Forms of retinal diseases with massive exudation. Roy Lond Ophthalmol Hosp Rep 1908; 17: 440-525.

2. Shields $\mathrm{CL}$, Udyaver S, Dalvin LA, et al. Coats disease in 351 eyes: Analysis of features and outcomes over 45 years (by decade) at a single center. Indian J Ophthalmol 2019; 67: 772-783.

3. Shields JA, Shields CL, Honavar SG, et al. Clinical variations and complications of Coats disease in 150 cases: The 2000 Sanford Gifford Memorial Lecture. Am J Ophthalmol 2001; 131:561-571.

4. Leber T. Ueber eine durch Vorkommen multipler Miliaraneurysmen charakterisierte Form von Retinaldegeneration. Albrecht von Graefe's Arch Klin Ophthalmol 1912; 81: 1-14.

5. Reese AB. Telangiectasis of the retina and Coats disease. Am J Ophthalmol 1956; 42: 1-8.

6. Daruich A, Matet A, Munier FL. Younger age at presentation in children with Coats disease is associated with more advanced stage and worse visual prognosis: A retirospective study. Retina 2018; 38: 2239-2246.

7. Lee A, Baek J, Ra H. A case of Leber's miliary aneurysms with diffuse peripheral retinal vascular sheathing. Indian J Ophthalmol 2018; 66: 1496-1498.

8. Querques G, Bux AV. Leber miliary aneurysms and multiple sclerosis. Eur J Ophthalmol 2009; 19: 690-693.

9. Knutsson KA, De Benedetto U, Querques G, et al. Pierwotne zaburzenia naczyniowe siatkówki: guzy i teleangiektazje. Okulistyka po Dyplomie 2012; 2: 45-55.

10. Turczyńska M, Brydak-Godowska J, Kęcik D. Obraz makrotętniak tętnicy siatkówki w badaniu 0CT. Okulistyka 2011; 14: 91-95.

11. Rabiolo A, Marchese A, Sacconi R, et al. Refining Coats' disease by ultra-widefield imaging and optical coherence tomography angiography. Graefes Arch Clin Exp Ophthalmol 2017; 255: 1881-1890.

12. Blair MP, Ulrich JN, Elizabeth Hartnett M, Shapiro MJ. Peripheral retinal nonperfusion in fellow eyes in coats disease. Retina 2013; 33: 1694-1699.

13. Calvo CM, Hartnett ME. The utility of ultra-widefield fluorescein angiography in pediatric retinal diseases. Int J Retina Vitreous 2018; 4: 21.

14. Sen M, Shields CL, Honavar SG, et al. Coats disease: An overview of classification, management and outcomes. Indian J Ophthalmol 2019; 67: 763-771.

15. Grosso A, Pellegrini M, Cereda MG, et al. Pearl and pitfalls in diagnosis and management of Coats disease. Retina 2015; 35: 614-623. 